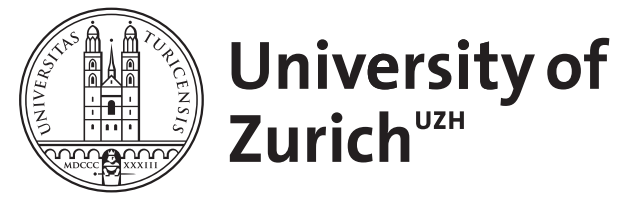

Zurich Open Repository and Archive

University of Zurich

University Library

Strickhofstrasse 39

CH-8057 Zurich

www.zora.uzh.ch

Year: 2018

Rare, yet relevant tumor cells - A new twist to melanoma cell plasticity

Zingg, Daniel ; Sommer, Lukas

DOI: https://doi.org/10.1111/pcmr.12643

Posted at the Zurich Open Repository and Archive, University of Zurich

ZORA URL: https://doi.org/10.5167/uzh-162701

Journal Article

Accepted Version

Originally published at:

Zingg, Daniel; Sommer, Lukas (2018). Rare, yet relevant tumor cells - A new twist to melanoma cell plasticity. Pigment Cell Melanoma Research, 31:7-10.

DOI: https://doi.org/10.1111/pcmr.12643 


\section{Rare, yet Relevant Tumor Cells - a New Twist to Melanoma Cell Plasticity.}

Daniel Zingg (orcid.org/0000-0001-6450-2534), Lukas Sommer (orcid.org/0000-0002-11437908)

Correspondence: Daniel Zingg (daniel.zingg@.uzh.ch), Lukas Sommer

(lukas.sommer@anatom.uzh.ch)

Metastatic cancers, including malignant cutaneous melanoma, have remained an incurable disease for most patients. Major advancements in the fields of targeted therapies and immunotherapies have brought some breakthrough to successfully eradicate metastatic melanoma. However, a large fraction of patients suffer from intrinsic or acquired resistances to these therapeutics and eventually display progressing disease (Hugo et al., 2015; Sharma et al., 2017; Sun et al., 2014). For many cancers, it is thought that intratumoral heterogeneity fosters tumor evolution. Tumor heterogeneity may arise from genetic aberrations as well as epigenetic mechanisms, such as DNA methylation or post-translational modifications of histones, both of which can heritably perturb gene expression (McGranahan and Swanton, 2017). Conceivably, during cancer therapies, resistances can emerge through an evolutionary process that selects pre-existing genetic or epigenetic sub-clones, the latter of which could consist of cancer stem-like cells. However, in an alternative scenario, cancer cells adaptively gain resistance mechanisms through either acquiring further genetic alterations or rewiring of the epigenome. Of note, both selection of resistant tumor sub-clones as well as plastic tumor cell behavior may be supported or even instructed by the microenvironment, especially the immune compartment (McGranahan and Swanton, 2017; Sharma et al., 2017). 
In recent years, small molecules targeting the MAPK signaling pathway, which is aberrantly activated in the majority of cutaneous melanomas, have emerged as therapies; for instance, the drug Vemurafenib is used to block the oncogenic activity of $\mathrm{BRAF}^{\mathrm{V} 600 \mathrm{E}}$. Comparing paired tumor biopsies before and after relapse to MAPK-blocking therapy revealed transcriptomic and underlying epigenetic alterations to be most recurrent, while de novo mutations were sparse (Hugo et al., 2015). Moreover, immunotherapies re-triggering cytotoxic T cells to eliminate tumor cells have recently stepped into the spotlight as promising melanoma treatments; Nivolumab, for instance, is a clinically applied antibody that blocks the negative immune checkpoint receptor PD-1 on $\mathrm{T}$ cells. Interestingly, similar to MAPK-blocking therapy, transcriptomic rather than genetic features define responses to anti-PD-1 immunotherapy (Sharma et al., 2017). Thus, apart from genetic perturbations, transcriptional changes and epigenomic rewiring appear to represent paradigm responses of melanoma cells to therapy. However, the data substantiating this idea have been gathered from bulk DNA and RNA sequencing. Hence, whether the observed transcriptional adaptations are a result of selecting epigenetically predefined sub-clones or of true tumor cell plasticity has remained unclear.

To shed light on this matter, Shaffer et al. elegantly took advantage of Luria and Delbrück's "fluctuation analysis", a method that was originally developed to study bacteria population heterogeneity versus mutation acquisition during gain of resistance to viral infections. In particular, the distribution of Vemurafenib-resistant clones emerging from a genetically homogeneous melanoma cell population was carefully analyzed. The data rejected the hypothesis that melanoma cells accumulate further mutations that would provide these cells with a selective advantage to acquire Vemurafenib resistance. To the contrary, the data strongly supported the hypothesis that any of the cells were capable of developing resistance to Vemurafenib, in favor of a cellular plasticity model. These findings are in agreement with the above-mentioned 
observations made in melanoma patients, which also found transcriptional and epigenetic changes to dominate resistance mechanisms (Hugo et al., 2015; Sharma et al., 2017; Sun et al., 2014).

Bulk RNA sequencing comparing melanoma cell cultures before and after Vemurafenib resistance then identified a differentially expressed set of genes, among which were $A X L, E G F R$, JUN, LOXL2, NGFR (CD271, p75 $\left.5^{\mathrm{NTR}}\right)$, and WNT5A. Importantly, all of these genes have previously been linked to both MAPK-targeted therapy resistance (Hugo et al., 2015; Sun et al., 2014) as well as inflammation, adoptive $\mathrm{T}$ cell transfer, or immunotherapy-induced melanoma immune escape (Hölzel and Tüting, 2016; Sharma et al., 2017; Zingg et al., 2017). Expression of aforementioned genes was low but not absent in cell cultures prior to Vemurafenib treatment. Therefore, Shaffer et al. screened single cells for the expression of resistance genes using singlemolecule RNA FISH and found a population of rare cells expressing these genes before drug exposure. When FACS-separating resistance marker-positive cells from marker-negative cells (e.g. NGFR ${ }^{+}$versus $\mathrm{NGFR}^{-}$), marker-positive cells were more likely to establish resistance upon Vemurafenib treatment. Notably, marker-negative cells were also capable to give rise to resistant colonies, though with a marginal frequency. Without exposure to Vemurafenib, however, resistance marker-positive cells lost resistance gene expression over time. Importantly, ATAC sequencing of marker-positive, pre-resistant cells revealed only little changes in chromatin accessibility in comparison to marker-negative, non-resistant cells. Conversely, cells underwent global chromatin rearrangements during acquisition of stable resistance, which also affected loci of resistance genes, such as EGFR. Moreover, promoters displaying chromatin compaction during resistance acquisition frequently shared a binding motif for SOX10, a melanocyte lineage transcription factor previously linked to Vemurafenib therapy resistance (Sun et al., 2014). In contrast, open chromatin regions were enriched for binding motives of transcription factors (e.g. AP-1, TEAD) regulating melanoma epithelial-mesenchymal transition (EMT) and invasion. Thus, a genetically homogeneous melanoma cell population contains cells that sporadically upregulate 
resistance genes, which renders these cells temporally pre-resistant. However, during Vemurafenib drug exposure, this poised resistance state becomes epigenetically engraved, resulting in stably reprogrammed resistant cells (Figure 1).

Shaffer et al. also found rare resistance marker-positive cells in human melanoma biopsies by taking advantage of both histological analyses and single-cell RNA sequencing data. However, in contrast to a controlled in vitro cell culture setup, cutaneous melanomas are genetically highly heterogeneous (McGranahan and Swanton, 2017). Thus, apart from reflecting sporadic in vivo gene expression of resistance genes, rare resistance marker-positive cells could, in principle, also belong to genetically distinct sub-populations. In murine melanoma models, adoptive $\mathrm{T}$ cell transfer and immunotherapies provoke tumor cell dedifferentiation, which includes epigenetic silencing of Sox10 and upregulation of resistance genes, such as Ngfr (Hölzel and Tüting, 2016; Zingg et al., 2017). Interestingly, this is dependent on the histone methyltransferase Ezh2, thus underpinning the relevance of adaptive epigenetic regulation, rather than clonal selection, for resistance acquisition to therapy (Zingg et al., 2017). Whether rare, pre-resistant cells, possibly with high Ezh2 activity, are also the dominant cell population to escape anti-tumor immunity, remains to be clarified.

Several of the resistance genes described by Shaffer et al. are well-established markers of melanoma dedifferentiation, stemness, and EMT. Interestingly, in murine melanoma, anti-tumor immunity and inflammation not only provoke $N g f r$ expression and melanoma stemness, but also metastatic spread (Hölzel and Tüting, 2016). Thus, it is plausible that the mechanism of poised resistance gene expression identified by Shaffer et al. not only renders melanoma effective in responding to targeted therapies and immunotherapy-induced immune pressure, but might also be implicated in EMT and metastasis formation in untreated patients. 


\section{Figure 1}

Human melanoma cells sporadically upregulate genes (e.g. $A X L, E G F R$, and NGFR) that have previously been linked to dedifferentiation, stemness, and EMT. Upon exposure to Vemurafenib melanoma therapy, cells temporally expressing such genes are more likely to develop Vemurafenib resistance as compared to cells negative for these genes. Therefore, sporadic expression of these genes renders melanoma cells pre-resistant to Vemurafenib. During resistance acquisition, global chromatin rearrangements, which include repression of SOX10-regulated genes and activation of dedifferentiation genes, then epigenetically stabilize the poised preresistant state resulting in stably reprogrammed resistant cells. The melanoma pre-resistance plasticity found by Shaffer et al. could conceivably be relevant for tumor immune responses as well as formation of metastasis. 


\section{References}

Hölzel, M., and Tüting, T. (2016). Inflammation-Induced Plasticity in Melanoma Therapy and Metastasis. Trends Immunol. 37, 364-374.

Hugo, W., Shi, H., Sun, L., Piva, M., Song, C., Kong, X., Moriceau, G., Hong, A., Dahlman, K.B., Johnson, D.B., et al. (2015). Non-genomic and Immune Evolution of Melanoma Acquiring MAPKi Resistance. Cell 162, 1271-1285.

McGranahan, N., and Swanton, C. (2017). Clonal Heterogeneity and Tumor Evolution: Past, Present, and the Future. Cell 168, 613-628.

Sharma, P., Hu-Lieskovan, S., Wargo, J.A., and Ribas, A. (2017). Primary, Adaptive, and Acquired Resistance to Cancer Immunotherapy. Cell 168, 707-723.

Sun, C., Wang, L., Huang, S., Heynen, G.J.J.E., Prahallad, A., Robert, C., Haanen, J., Blank, C., Wesseling, J., Willems, S.M., et al. (2014). Reversible and adaptive resistance to BRAF(V600E) inhibition in melanoma. Nature 508, 118-122.

Zingg, D., Arenas-Ramirez, N., Sahin, D., Rosalia, R.A., Antunes, A.T., Haeusel, J., Sommer, L., and Boyman, O. (2017). The Histone Methyltransferase Ezh2 Controls Mechanisms of Adaptive Resistance to Tumor Immunotherapy, Cell Reports 20, 854-867. 


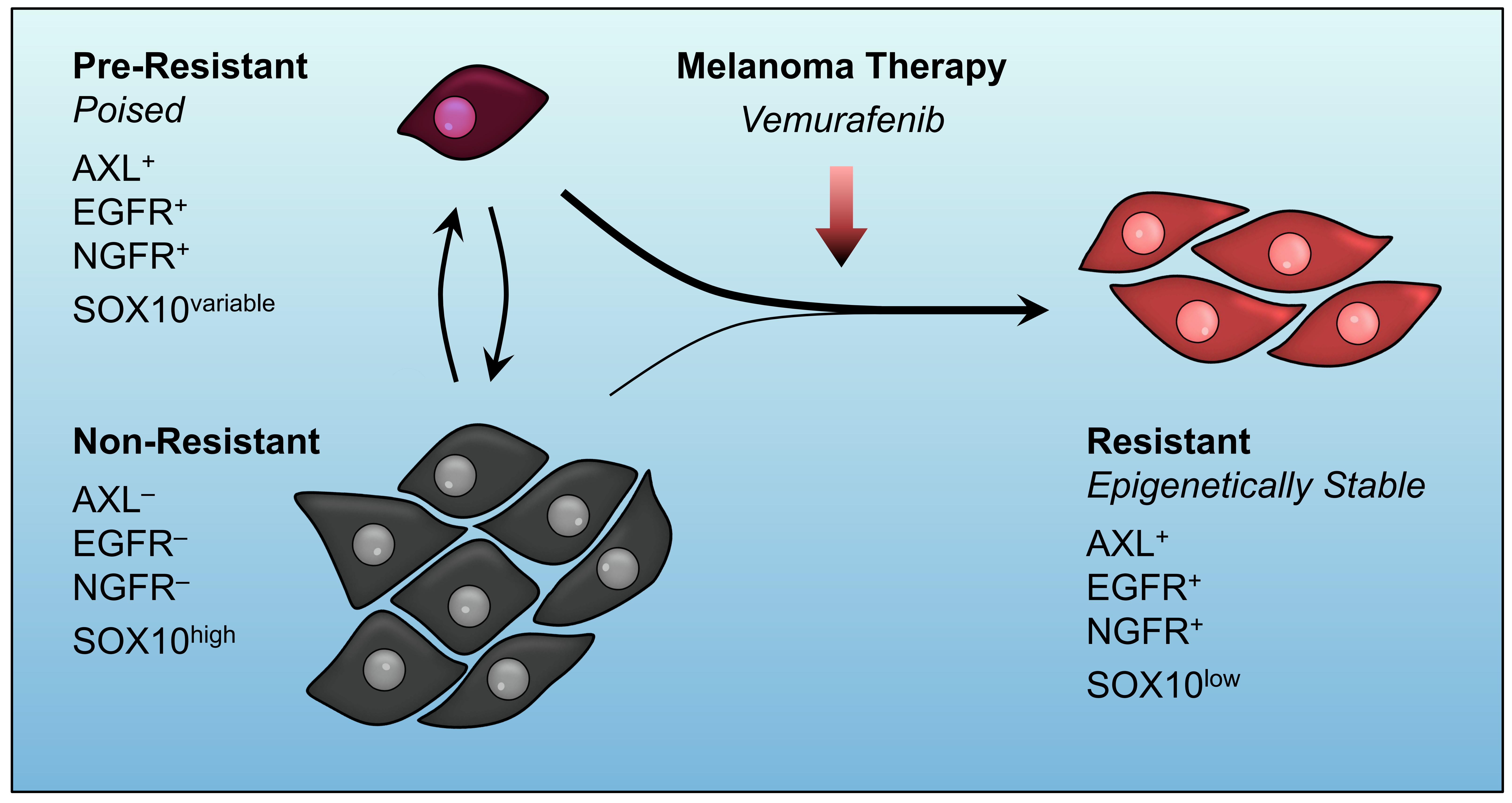

